\section{Recent advances in local anaesthesia}

In recent years the major interest and advances in local anaesthesia have involved investigations regarding the relative cardiovascular and localized neural effects of local anaesthetic agents. In general, local anaesthetic agents are relatively free of side effects if they are administered in an appropriate dosage and in the appropriate anatomical location. The vast majority of toxic reactions occur following the accidental intravascular or subarachnoid injection of a large dose of a local anaesthetic. In addition, the administration of an excessive dose into the appropriate anatomical location can lead to systemic toxicity following the vascular absorption of the local anaesthetic agent. Since the introduction of cocaine in 1884 , both systemic and local toxic reactions have been reported following the use of local anaesthetic agents.

\section{Comparative cardiovascular effects of local anaesthetics}

Most of the early studies concerning the cardiodepressant effects of local anaesthetics indicated that a relationship exists between the intrinsic anaesthetic potency of the various agents and their relative negative inotropic activity. In recent years there has been some suggestion that the more potent local anaesthetic agents such as bupivacaine may be relatively more cardiotoxic than the less potent local anaesthetics, such as lidocaine. Several case reports have appeared in the literature in which bupivacaine and etidocaine were associated with rapid and profound cardiovascular depression. These cases differed from the usual cardiovascular depression seen with local anaesthetics. The onset of cardiovascular depression occurred relatively early. In some cases, severe cardiac arrhythmias were observed, and the cardiac depression appeared resistant to various therapeutic modalities.

The cardiotoxicity of the more potent agents such as bupivacaine appears to differ from that of lidocaine in the following manner: (1) The ratio of the dosage required for irreversible cardiovascular collapse and the dosage which will produce CNS toxicity (convulsions), i.e., the CC/CNS ratio, is lower for bupivacaine and etidocaine compared to lidocaine. (2) Ventricular arrhythmias and fatal ventricular fibrillation may occur following the rapid intravenous administration of a large dose of bupivacaine but not lidocaine. (3) The pregnant animal or patient may be more sensitive to the cardiotoxic effects of bupivacaine than the non-pregnant animal or patient. (4) Cardiac resuscitation is more difficult following bupivacaine induced cardiovascular collapse. (5) Acidosis and hypoxia markedly potentiate the cardiotoxicity of bupivacaine.

\section{CC/CNS ratio}

The dosage and blood level of lidocaine, bupivacaine and etidocaine associated with the development of convulsive activity and cardiovascular collapse has been determined in adult sheep. A $\mathrm{CC} / \mathrm{CNS}$ dose ratio of $7.1 \pm 1.1$ existed for lidocaine indicating that seven times as much drug was required to induce irreversible cardiovascular collapse as was needed for the production of convulsions. In comparison, the $\mathrm{CC} / \mathrm{CNS}$ ratio for bupivacaine was $3.7 \pm 0.5$ and for etidocaine $4.4 \pm 0.9$. Thus, although the central nervous system was still more sensitive to the toxic effects of the more potent local anaesthetics, a smaller difference did exist between the dose to cause convulsions and that which led to irreversible cardiovascular collapse as compared to the less potent local anaesthetic, lidocaine.

Studies in sheep in which the blood levels of various local anaesthetics were determined and related to the onset of CNS and cardiovascular toxicity also reveal a narrower $\mathrm{CC} / \mathrm{CNS}$ ratio for the more potent local anaesthetics. For example, lidocaine was found to possess a CC/CNS blood level ratio of $3.6 \pm 0.3$ compared to values of 1.6 to 1.7

Department of Anaesthesia, Harvard Medical School, and Department of Anesthesia, Brigham and Women's Hospital, 75 Francis St., Boston, MA 02115. 
for bupivacaine and etidocaine. Tissue levels of the various local anaesthetics determined at the time of cardiovascular collapse indicate a greater uptake of bupivacaine and etidocaine by the myocardium compared to lidocaine. Thus, the enhanced sensitivity of the myocardium to these more potent agents appears to be due to a greater myocardial uptake.

\section{Ventricular arrhythmias}

A number of investigators have reported the development of ventricular arrhythmias in animals exposed to toxic doses of bupivacaine. Bupivacaine was reported to cause cardiac arrhythmias in awake but paralyzed cats, whereas no such changes were observed with lidocaine. Studies in unanaesthetized sheep demonstrated that severe cardiac arrhythmias occurred following the rapid intravenous administration of bupivacaine but not lidocaine. Studies in unanaesthetized dogs demonstrated the occurrence of ventricular tachycardia and ventricular fibrillation in some of the animals receiving intravenous bupivacaine, but no arrhythmias occurred when the same dogs were given intravenous lidocaine.

It is not known whether the occurrence of ventricular arrhythmias is a toxic effect related to bupivacaine alone or may be produced by other local anaesthetics. In a preliminary study in awake dogs convulsant and supraconvulsant doses of lidocaine, mepivacaine, bupivacaine, and etidocaine were administered intravenously. Ventricular fibrillation occurred in approximately 50 per cent of dogs following the rapid intravenous injection of a convulsant or supraconvulsant dose of bupivacaine. Ventricular fibrillation was not observed in the lidocaine-, mepivacaine- or etidocaine-treated dogs. However, in a previous study one of four dogs which received $10 \mathrm{mg} \cdot \mathrm{kg}^{-1}$ of etidocaine did develop ventricular fibrillation. In addition, ventricular arrhythmias were observed in awake paralyzed cats following the administration of etidocaine, although the frequency was less than that associated with the use of bupivacaine. The results suggest that the occurrence of ventricular fibrillation is not related to the basic piperidine ring structure of bupivacaine since mepivacaine which contains the piperidine moeity failed to cause these cardiac abnormalities. In addition, a precise correlation does not appear to exist between the frequency of ventricular arrhythmias and the lipid solubility and protein binding of local anaestheics. Large doses of etidocaine, which is more lipid-soluble than bupivacaine and equally protein-bound, may cause ventricular arrhythmias and fibrillation but the incidence appears to be lower than that observed with bupivacaine.

The aetiology of bupivacaine associated ventricular arrhythmias has been investigated. Electrophysiological studies on isolated tissues have shown that bupivacaine can markedly depress the rapid phase of depolarization $\left(V_{\max }\right)$ of the cardiac action potential. The recovery phase is prolonged in isolated papilary muscles, and conduction block and electrical inexcitability were observed in canine Purkinje fibres exposed to bupivacaine suggesting the possibility of unidirectional blockade of conducting pathways leading to a re-entrant type of arrhythmia.

\section{Enhanced cardiotoxicity in pregnancy}

It is not certain whether the pregnant patient is more susceptible to the toxic effects of local anaesthetics. The CC/CNS dosage ratio decreased from $3.7 \pm$ 0.5 in non-pregnant sheep to $2.7 \pm 0.4$ in pregnant animals. However, little difference was observed in the CC/CNS blood level ratio which varied from $1.6 \pm 0.1$ in non-pregnant animals to $1.4 \pm 0.1$ in pregnant ewes. However, the blood level of bupivacaine which resulted in cardiovascular collapse was lower in pregnant animals. Those studies in sheep suggest that the pregnant animals may be more sensitive to the cardiotoxic effects of bupivacaine. However, it is not known whether this enhanced vulnerability is unique to bupivacaine or may be common to all local anaesthetic agents.

\section{Cardiac resuscitation}

Cardiopulmonary resuscitation is reportedly diffcult in some patients in whom cardiotoxicity has occurred following the administration of a toxic dose of bupivacaine. Studies in acidotic and hypoxic sheep have also indicated that cardiac resuscitation following bupivacaine induced toxicity is difficult. However, recent studies in hypoxic dogs rendered toxic with bupivacaine indicate that resuscitation is possible if massive doses of epinephrine and atropine are employed. Moreover, investigations in cats revealed little difference in the ability to resuscitate animals rendered cardiotoxic with either lidocaine or bupivacaine. 


\section{Effect of acidosis and hypoxia}

Changes in acid-base status will alter the potential cardiovascular toxicity of local anaesthetic agents. Studies on isolated atrial tissues have shown that hypercarbia, acidosis and hypoxia will tend to potentiate the negative chronotropic and inotropic action of lidocaine and bupivacaine. In particular, the combination of hypoxia and acidosis appears to markedly potentiate the cardiodepressant effects of bupivacaine. Studies in intact sheep have also demonstrated that hypoxia and acidosis markedly increase the frequency of cardiac arrhythmias following the intravenous administration of bupivacaine and also increase the mortality rate. Enhanced toxicity in the presence of acidosis does not appear related to a greater myocardial tissue uptake of local anaesthetic since investigations in rabbits demonstrated a decreased cardiac concentration of bupivacaine in the presence of acidosis. Marked hypercarbia, acidosis and hypoxia occur very rapidly in some patients following seizure activity due to the rapid accidental intravascular injection of local anaesthetic agents. Thus, the cardiovascular depression may be related in part to the severe acid-base changes that occur following the administration of toxic doses of these agents.

\section{Local tissue toxicity}

Although the primary toxic effect of local anaesthetic agents involves the systemic administration of excessive doses of these drugs, the potential local irritant action of this class of compounds has also been the subject of considerable interest. Local anaesthetic agents which are employed clinically rarely produce localized nerve damage. Studies on isolated frog sciatic nerve revealed that concentrations of procaine, cocaine, tetracaine, and dibucaine required to produce irreversible conduction blockade are far in excess of the concentration of these agents used clinically. Recently, however, some concern has been expressed regarding the potential neurotoxicity of chloroprocaine. The possibility that this agent can cause localized neural damage is based on the report of prolonged sensory and motor deficits in four patients following the epidural or subarachnoid injection of large doses of this particular drug. Subsequently, Moore reported signs of neural damage in five additional patients in whom chloroprocaine had been employed. Studies in animals have proven somewhat contradictory regarding the potential neurotoxicity of chloroprocaine. For example, data from isolated rabbit vagus nerve preparations indicated that chloroprocaine was associated with signs of neural irritation, whereas the use of lidocaine under similar conditions failed to cause local toxic effects. However, histological examination of rabbit sciatic nerves exposed to chloroprocaine for a period of six hours did not reveal any signs of nerve damage. When doses of several local anaesthetics sufficient to cause total spinal anaesthesia in dogs were employed, chloroprocaine produced total paralysis in approximately 30 per cent of the animals, whereas none of bupivacaine-treated dogs showed evidence of permanent neurological sequelae. Studies of a similar nature in sheep and monkeys failed to show any difference in neurotoxicity between chloroprocaine and other local anaesthetics or control solutions. More recently, it has been reported that paralysis was observed in rabbits in whom chloroprocaine solutions were administered intrathecally. However, the paralysis was believed to be related to the sodium bisulfite which is employed as an antioxidant in chloroprocaine solutions. The use of pure solutions of chloroprocaine without sodium bisulfite did not cause paralysis whereas the sodium bisulfite alone was associated with paralysis. A detailed series of studies has been conducted on the isolated rabbit vagus nerve to investigate the neurotoxicity of the various components of commercial chloroprocaine solutions. Commercial solutions of three per cent chloroprocaine contain the local anaesthetic agent itself, 0.2 per cent sodium bisulfite and hydrogen ions which yield a $\mathrm{pH}$ of approximately 3.0. Application of commercial three per cent chloroprocaine to isolated vagus nerves for 30 minutes resulted in irreversible conduction blockade. The use of a three per cent chloroprocaine with sodium bisulfite solution buffered to a pH of 7.0 caused reversible conduction block. A three per cent chloroprocaine solution with a $\mathrm{pH}$ of 3.0 but without sodium bisulfite also resulted in reversible blockade. Application of an 0.2 per cent sodium bisulfite solution at a $\mathrm{pH}$ of 3.0 resulted in irreversible conduction block whereas the use of a 0.2 per cent sodium bisulfite solution with a $\mathrm{pH}$ of 7.0 caused no conduction block. The results of these studies suggest that the combination of a low $\mathrm{pH}$ and the presence of sodium bisulfite may be responsible for the neurotoxic reactions 
observed following the use of large amounts of chloroprocaine solution. Chloroprocaine itself does not appear to be neurotoxic.

\section{Summary}

In general, the cardiovascular system is more resistant to the toxic actions of local anaesthetics than is the central nervous system. However, if sufficient doses and blood levels of local anaesthetics are achieved, signs of profound cardiovascular depression may be observed. Differences exist between local anaesthetics in terms of their relative potential for cardiotoxicity. The CC/CNS ratio for bupivacaine and etiodcaine is less than for lidocaine. In addition, bupivacaine may precipitate ventricular arrhythmias and ventricular fibrillation.

Local tissue toxicity can occur following the administration of local anaesthetics. In general, neural tissue appears to be relatively resistant to the irritant effects of local anaesthetic drugs. However, large dosages of chloroprocaine solutions administered intrathecally have been associated with prolonged sensory-motor deficits in a few patients due probably to the low $\mathrm{pH}$ and presence of sodium bisulfite in the chloroprocaine solutions.

In general, the incidence of toxic reactions to local anaesthetic agents is extremely low. However, as with any class of pharmacological agents, local anaesthetics may cause severe toxic reations, due usually to the improper use of these drugs.

\section{Suggested reading}

1 Clarkson CW, Hohdeghem LM. Mechanism for bupivacaine depression of cardiac conduction: fast block of sodium channels during the action potential with slow recovery from block during diastole. Anesthesiology 1985; 62: 396-405.

2 Morishima HO, Pederson H, Finster $M$ et al. Bupivacaine toxicity in pregnant and nonpregnant ewes. Anesthesiology 1985; 63: 134-9.

3 Kotelko DM, Shnider SM, Dailey PA et al. Bupivacaine-induced cardiac arrhythmias in sheep. Anesthesiology 1984; 60: 10-8.

4 Kasten GW, Martin ST. Bupivacaine cardiovascular toxicity: comparison of treatment with bretylium and lidocaine. Anesth Analg 1984; 64: 911-6.

5 Ravindran RS, Bond VK, Tasch MD, Gupra CD Luerssen TG. Prolonged neural blockade following regional analgesia with 2-chloroprocaine. Anesth Analg 1980; 58: 447-51.

6 Wang BC, Hillman DE, Spiedholz NI, Turndorf $H$. Chronic neurological deficits and Nesacaine-CE - an effect of the anesthetic, 2-chloroprocaine, or the antioxidant, sodium bisulfite? Anesth Analg 1984; 63: 445-7.

7 Gissen AJ, Datta S, Lambert D. The chloroprocaine controversy II: is chloroprocaine neurotoxic? Reg Anesth 1984; 9: 135-45. 